\title{
An Inequalities for the Trace of the Block Hadamard Product
}

\author{
Hui Quan* \\ Department of mathematics, Xiangtan University, Xiangtan 411105, China
}

*Corresponding Author: Hui Quan, Department of mathematics, Xiangtan University, Xiangtan 411105, China

Abstract: Let $\lambda_{1}(A) \geq \ldots \geq \lambda_{n}(A)$ denote the eigenvalues of a Hermitiann by $n$ matrix $A$, our main results are $\log \lambda\left(C^{*} C\right) \mathrm{p}_{w} \log \lambda(A) \mathrm{o} \lambda(B)$ and $\lambda\left(C^{*} C\right) \mathrm{p}_{w} \lambda(A) \mathrm{o} \lambda(B)$. Here $A$ is a positive matrix, $B$ and $M=\left[\begin{array}{cc}A & C^{*} \\ C & B\end{array}\right]$ are positive semi-definite Hermitian matrices.

Keywords: Eigenvalue, Positive Semi-definite Matrix, Majorization

\section{INTRODUCTION}

Let $A$ and $B$ be positive semi-definite. The following results due to Boying Wang and Fuzhen Zhang [1] says that $\prod_{j=1}^{k} \lambda_{t}(A B) \geq \prod_{j=1}^{k} \lambda_{i_{t}}(A) \lambda_{n-i_{t}+1}(B)$ with $1 \leq i_{1}<\ldots<i_{k} \leq n$. Gunther and Klotz presented a survey focusing on the study of a block Hadamard and block Kronecker products of positive semidefinite matrices in 2012[3]. Saliha Pehlivan [4] provided some trace inequalities for the trace of the block Hadamard product. In this paper, we shall be mainly interested in the inequalities for the trace of the block Hadamard product.

\section{PReliminaries}

Let $C^{n \times n}$ denote the vector space of all $n$ by $n$ complex matrices. Denote by $\lambda_{1}(A) \geq \ldots \geq \lambda_{n}(A)$ the eigenvalues of a Hermitian matrix $A \in C^{n \times n}$.

Let $x=\left(x_{1}, x_{2}, \ldots, x_{n}\right)^{T}, y=\left(y_{1}, y_{2}, \ldots, y_{n}\right)^{T}$ with $x_{1} \geq x_{2} \geq \ldots \geq x_{n}, y_{1} \geq y_{2} \geq \ldots \geq y_{n}$.If

$\sum_{i=1}^{k} x_{i} \leq \sum_{i=1}^{k} y_{i}, k=1,2, \ldots, n$, we say that $x$ is weakly majorized by $y$,and write $x \mathrm{p}_{w} y$.

If $\prod_{i=1}^{k} x_{i} \leq \prod_{i=1}^{k} y_{i}, k=1,2, \ldots, n$, we write $x \mathrm{p}_{w \log } y$, or $\log x \mathrm{p}_{w} \log y$.

\section{Main Results}

We start with the following known results.

Lemma1.1 If $A, B \in C^{n \times n}$ be Hermitian, then for any $1 \leq j \leq n$,

we have $\max _{r+s=j+n}\left\{\lambda_{r}(A)+\lambda_{s}(B)\right\} \leq \lambda_{j}(A+B) \leq \min _{r+s=j+1}\left\{\lambda_{r}(A)+\lambda_{s}(B)\right\}$.

Lemma1.2 If $A, B$ are the same order matrix, the $A B$ and $B A$ have the same non-zero eigenvalues. Similarly, the same order matrix $A, B, C, A B C$ and $C A B$ also have the same non-zero eigenvalues.

Lemma1.3 If $A, B \in C^{n \times n}$ are positive semi-definite Hermitian and $1 \leq i_{1}<\ldots<i_{k} \leq n$, then 
$\prod_{j=1}^{k} \lambda_{t}(A B) \geq \prod_{j=1}^{k} \lambda_{i_{t}}(A) \lambda_{n-i_{t}+1}(B)$ with equality for $k=n$

Lemma1.4 For $\quad x, y \in R_{+}^{n}, \prod_{j=1}^{k} x_{j}^{\downarrow} \leq \prod_{j=1}^{k} y_{j}^{\downarrow}, 1 \leq k \leq n \Rightarrow \sum_{j=1}^{k} x_{j}^{\downarrow} \leq \sum_{j=1}^{k} y_{j}^{\downarrow}, 1 \leq k \leq n$. Where $x_{j}^{\downarrow}, y_{j}^{\downarrow}$ means reorder $x_{1}, x_{2}, \mathrm{~L}, x_{\mathrm{k}}$ and $y_{1}, y_{2}, \mathrm{~L}, y_{\mathrm{k}}$ from smallest to largest.

Theorem $1 A, B, C$ are square matrices of order n, with $A$ being positive definite matrix, $B$ and $M=\left[\begin{array}{cc}A & C^{*} \\ C & B\end{array}\right]$ are positive semi-definite matrices,

then $\log \lambda\left(C^{*} C\right) \mathrm{p}_{w} \log \lambda(A) \mathrm{o} \lambda(B), \quad \lambda\left(C^{*} C\right) \mathrm{p}_{w} \lambda(A) \mathrm{o} \lambda(B)$.

Proof Considered contract changes

$\left[\begin{array}{cc}I & -A^{-1} C^{*} \\ 0 & I\end{array}\right]^{*}\left[\begin{array}{cc}A & C^{*} \\ C & B\end{array}\right]\left[\begin{array}{cc}I & -A^{-1} C^{*} \\ 0 & I\end{array}\right]=\left[\begin{array}{cc}A & 0 \\ 0 & B-C A^{-1} C^{*}\end{array}\right]$

Due to $M=\left[\begin{array}{cc}A & C^{*} \\ C & B\end{array}\right]$ is a positive definite matrix, from the properties of positive definite matrix we can know that $B-C A^{-1} C^{*} \geq 0$, in other words $B \geq C A^{-1} C^{*}$, so, from lemma 1.1 we have $\lambda_{j}(B) \geq \lambda_{j}\left(C A^{-1} C^{*}\right)$, from lemma 1.2 we have

$\lambda_{j}\left(C A^{-1} C^{*}\right)=\lambda_{j}\left(C^{*} C A^{-1}\right)$, thus $\lambda_{j}(B) \geq \lambda_{j}\left(C^{*} C A^{-1}\right)$. from lemma 1.3 we know that

$\prod_{j=1}^{k} \lambda_{j}(B) \geq \prod_{j=1}^{k} \lambda_{j}\left(C^{*} C A^{-1}\right) \geq \prod_{j=1}^{k} \lambda_{j}\left(C^{*} C\right) \lambda_{n-j+1}\left(A^{-1}\right), 1 \leq k \leq n$

Due to $\lambda_{n-j+1}\left(A^{-1}\right)=\frac{1}{\lambda_{j}(A)}$, we can know $\prod_{j=1}^{k} \lambda_{j}(B) \lambda_{j}(A) \geq \prod_{j=1}^{k} \lambda_{j}\left(C^{*} C\right), 1 \leq k \leq n$. So we can prove that $\lambda\left(C^{*} C\right) \mathrm{p}_{w \log } \lambda(A) \mathrm{o} \lambda(B)$. Obviously $\prod_{j=1}^{k} \lambda_{j}(B) \lambda_{j}(A)=\prod_{j=1}^{k} \lambda^{\downarrow}{ }_{j}(B) \lambda_{j}^{\downarrow}(A)$ $\prod_{j=1}^{k} \lambda_{j}\left(C^{*} C\right)=\prod_{j=1}^{k} \lambda_{j}^{\downarrow}\left(C^{*} C\right)$, then we have $\prod_{j=1}^{k} \lambda_{j}^{\downarrow}(B) \lambda^{\downarrow}{ }_{j}(A) \geq \prod_{j=1}^{k} \lambda_{j}^{\downarrow}\left(C^{*} C\right), 1 \leq k \leq n$. from lemma 1.4 we know that $\sum_{j=1}^{k} \lambda^{\downarrow}{ }_{j}(A) \lambda^{\downarrow}{ }_{j}(B) \geq \sum_{j=1}^{k} \lambda_{j}^{\downarrow}\left(C^{*} C\right), 1 \leq k \leq n$. So we can get that $\lambda\left(C^{*} C\right) \mathrm{p}_{w} \lambda(A) \mathrm{o} \lambda(B)$.

From $\lambda\left(C^{*} C\right) \mathrm{p}_{w \log } \lambda(A) \mathrm{o} \lambda(B)$ we can get that $\lambda\left(C^{*} C\right)^{p} \mathrm{p}_{w \log } \lambda\left(A^{p}\right) \mathrm{o} \lambda\left(B^{p}\right)$, where $0<p<1$. In particular, when $p=\frac{1}{2}$, we can also get $\delta(C) \mathrm{p}_{\text {wlog }} \lambda\left(A^{\frac{1}{2}}\right) \mathrm{o} \lambda\left(B^{\frac{1}{2}}\right)$, and then we can get $\delta(C) \mathrm{p}_{w} \lambda\left(A^{\frac{1}{2}}\right) \mathrm{o} \lambda\left(B^{\frac{1}{2}}\right)$. When $k=n, \prod_{j=1}^{n} \lambda_{j}(B) \lambda_{j}(A) \geq \prod_{j=1}^{n} \lambda_{j}\left(C^{*} C\right)$, we can know that $\operatorname{det} A \operatorname{det} B \geq \operatorname{det}\left(C^{*} C\right)$.

\section{Note}

we know that when $A, B \geq 0, \lambda(A \circ B) \mathrm{p}_{w} \lambda(A) \mathrm{o} \lambda(B)$ is true, but the inequality in the above theorem cannt be strengthened to $\lambda\left(C^{*} C\right) \mathrm{p}_{w} \lambda(A \circ B)$. 
Here is a example :

Take $A=\left[\begin{array}{ll}1 & 0 \\ 0 & 2\end{array}\right], B=\left[\begin{array}{cc}3 & 2 \\ 2 & 1.6\end{array}\right], C=\left[\begin{array}{ll}1 & 1 \\ 2 & 1\end{array}\right]$, it can be calculated that $\operatorname{tr}\left(C^{*} C\right) \geq \operatorname{tr}(A \circ B)$.

Corollary $1 A, B, C, M$ conditions are the same as above, the $\operatorname{tr} A \operatorname{tr} B \geq \operatorname{tr}\left(C^{*} C\right)$.

Corollary 2 Let $A, B$ are n-order Hermitematrix, and the block matrix $\left[\begin{array}{cc}A & C^{*} \\ C & B\end{array}\right] \geq 0, B \geq 0$, then $\delta(C) \mathrm{p}_{w \log } u, \quad$ where $u=\left(u_{1}, u_{2}, \ldots, u_{n}\right), \quad u_{i}=\max \left\{\lambda_{i}(A), \lambda_{i}(B)\right\}$

\section{REFERENCES}

[1] Wang B, Zhang F. Some inequalities for the eigenvalues of the product of positive semidefinite Hermitian matrices [J]. Linear Algebra and Its Applications, 1992, 160(1):113-118.

[2] Wang B Y, Gong M P. Some eigenvalue inequalities for positive semidefinite matrix power products[J]. Linear Algebra \& Its Applications, 1993, 184(2):249-260.

[3] M. Gunther, L. Klotz, Schur'z theorem for a block Hadamard product, Linear Algebra and its Applications, 437(2012) 948-956.

[4] Saliha Pehlivan, Trace Inequalities for a Block Hadamard Product, Filomat. (2018), 285-292

Citation: Hui Quan, (2019). An Inequalities for the Trace of the Block Hadamard Product. International Journal of Scientific and Innovative Mathematical Research (IJSIMR), 7(7), pp.1-3. http://dx.doi.org/ 10.20431/2347-3142.0706001

Copyright: () 2019 Authors, this is an open-access article distributed under the terms of the Creative Commons Attribution License, which permits unrestricted use, distribution, and reproduction in any medium, provided the original author and source are credited. 